\title{
Effects of frequent multiple plateletpheresis on complete blood count values of blood donors
}

\author{
Jun $\mathrm{Li}^{1}$, Hongmei Liao ${ }^{2 *}$ \\ ${ }^{1}$ Department of Apheresis Component, Chongqing Blood Center, Chongqing 400015, China; \\ ${ }^{2}$ The Institute of Blood Transfusion, Chongqing Blood Center, Chongqing 400015, China.
}

\begin{abstract}
The use of apheresis technique to collect platelets has rapidly increased in recent years. With an increased demand for plateletpheresis, higher donation frequencies are now observed. The aim of this study is to investigate changes in complete blood count values after frequent multiple plateletpheresis. A total of seventy-four blood donors were selected, from which complete blood count values in the first and the last screening were taken. There were fifty-four high frequency donors(13.0 \pm 2.6 plateletpheresis/year) and twenty low frequency donors( $6.6 \pm 0.5$ plateletpheresis/year). The results showed that complete blood count values at the first and last screening after plateletpheresis of both groups were within the normal range, and changes in their WBC, RBC and PLT values were not statistically significant $(P>0.05, P>0.05$, and $P>0.05$, respectively). The study suggests that frequent multiple plateletpheresis has no effects on complete blood count, and no adverse effects on blood donors.
\end{abstract}

Keywords: plateletpheresis; blood donors; blood routine

\section{INTRODUCTION}

The blood is made up of many cells, most of which are red blood cells, white blood cells and platelets. Platelet transfusions play an important role in the prevention or treatment of bleeding in patients with thrombocytopenia or severely impaired platelet function. Platelets obtained from apheresis technique are termed as plateletpheresis ${ }^{[1]}$. The efficiency of plateletpheresis has been improving owing to new developments in automated cell separators in the past years. Plateletpheresis is generally a safe procedure for platelet donation ${ }^{[2]}$, and is rapidly growing in the blood collection field. Because of its advantages ${ }^{[3]}$,

*Correspondence to: Hongmei Liao, The Institute of Blood Transfusion, Chongqing Blood Center, 1 Guihuayuan Road, Yuzhong District, Chongqing 400015, China. E-mail: 41232956@foxmail.com Conflict of interests: The authors state that there is no conflict of interests associated with this publication. plateletpheresis is receiving increasing attention and is currently in widespread clinical use. However, due to a variety of other factors, such as serious adverse reactions ${ }^{[4]}$, plateletpheresis donors face a variety of difficulties compared to donors who donate whole blood. So while the demand for plateletpheresis has been increasing, the provision of voluntary and eligible blood donors is becoming more and more difficult. In general, a plateletpheresis donor must meet the requirements of a whole blood donor. However more stringent assessments are required for donors who participate in more frequent donations. Therefore, how to retain frequent multiple plateletpheresis donors while ensuring safety is very important for maintaining clinical supply.

The effect of plateletpheresis on haematopoietic system remains significant. According to some reports, plateletpheresis has an adverse effect on donor haematopoiesis with short term and long term effects like anemia, thrombocytopenia, lymphocytopenia ${ }^{[5]}$. 
After multiple plateletpheresis, patents' recovery to their pre-plateletpheresis status must be monitored by medics in the blood collection field.

To evaluate the effects of plateletpheresis on complete blood count values in donors, we performed a statistical analysis. This study should help to ensure the safety of plateletpheresis donors, as well as to provide reference for the development of safety standards.

\section{MATERIALS AND METHODS}

\section{Blood donors}

Standard procedures(GB18467-2011) derived from National Health Commission guidelines for plateletpheresis donor selection was followed. Seventy-four blood donors were included in the study. Standard guidelines were followed: age range from 18 to 60 years; weight of females and males: not less than $45 \mathrm{~kg}$ and $50 \mathrm{~kg}$; hemoglobin concentration: at least $115 \mathrm{~g} / \mathrm{L}$ for females and $120 \mathrm{~g} / \mathrm{L}$ for males; platelet count: $\geqslant 150 \times 10^{9} / \mathrm{L}$ and $<450 \times 10^{9} / \mathrm{L} ; \mathrm{ALT}$ : no more than $50 \mathrm{U} / \mathrm{L}$. The study was approved by the Institutional Ethics Committee of Chongqing Blood Center. Informed consent was obtained from all individual participants included in the study.

\section{Complete blood screening}

Firstly, pre-plateletpheresis donors were requested to fill the donor questionnaire form. Secondly, pre- plateletpheresis donors were subjected to a clinical examination. Lastly, peripheral blood samples $(2 \mathrm{~mL}$, EDTA) from each pre-plateletpheresis donor was sent for blood routine testing. Complete blood counts were determined by automated blood cell counter (Medonic M-series, Sweden).

\section{Statistical analysis}

Complete blood count values from the first and the last screening were collected and analyzed. For normally distributed variables, the results were expressed as Mean \pm SD. The values were compared using the paired t test, and $P<0.05$ was considered as statistically significant. Statistical analysis was done with GraphPad Prism 6 (GraphPad Software, USA) and Excel 2010 (Microsoft Corporation, USA).

\section{RESULTS}

\section{Post plateletpheresis results from the high frequency group}

In Table 1 3, statistical comparisons from complete blood count data (from the first and the last plateletpheresis) in the high frequency group $(n=54$; frequency $=13.0 \pm 2.6$ per year) are shown. After plateletpheresis, WBC, RBC and PLT values obeyed normal distribution, and all hematological parameters were within the normal range and showed no significant change $(P>0.05)$.

Table 1 PLT count values for the first and the last screening in high frequent group $(n=54)$

\begin{tabular}{cccccc}
\hline Time & PLT* $^{*}()$ & MPV* $\left.^{*}\right)$ & PDW*$\left.^{*}\right)$ & PCT $^{*}()$ & LPCR*( ) \\
\hline First & $222.72 \pm 48.05$ & $7.64 \pm 0.83$ & $11.28 \pm 1.19$ & $0.16 \pm 0.03$ & $13.51 \pm 5.09$ \\
Last & $223.76 \pm 55.95$ & $7.61 \pm 0.98$ & $11.33 \pm 1.37$ & $0.17 \pm 0.03$ & $13.59 \pm 5.80$ \\
\hline
\end{tabular}

Note: ${ }^{*} P>0.05$

Table 2 WBC count values for the first and the last screening in high frequent group $(n=54)$

\begin{tabular}{|c|c|c|c|c|c|c|c|}
\hline Time & WBC*( ) & LA*( ) & MA*( ) & GA*( ) & $\mathrm{LR}^{*}(\mathrm{)}$ & $\mathrm{MR} *(\quad)$ & $\mathrm{GR}^{*}(\mathrm{)})$ \\
\hline First & $6.24 \pm 1.34$ & $1.66 \pm 0.44$ & $0.49 \pm 0.12$ & $4.09 \pm 1.18$ & $27.79 \pm 6.63$ & $7.13 \pm 1.31$ & $65.08 \pm 7.46$ \\
\hline Last & $6.14 \pm 1.40$ & $1.70 \pm 0.5$ & $0.48 \pm 0.12$ & $3.95 \pm 1.15$ & $28.78 \pm 6.93$ & $7.12 \pm 0.99$ & $64.11 \pm 7.34$ \\
\hline
\end{tabular}

Note: ${ }^{*} P>0.05$

Table 3 RBC count values for the first and the last screening in high frequent group ( $n=54)$

\begin{tabular}{|c|c|c|c|c|c|c|c|c|}
\hline Time & RBC*( ) & $\mathrm{HGB}^{*}(\mathrm{)}$ & HCT*( ) & $\mathrm{MCV}^{*}(\mathrm{)}$ & $\mathrm{MCH}^{*}(\mathrm{)}$ & MCHC*( ) & RDWR*( ) & RDWA*( ) \\
\hline First & $4.85 \pm 0.35$ & $144.04 \pm 8.89$ & $43.48 \pm 2.79$ & $89.94 \pm 5.79$ & $29.83 \pm 2.08$ & $319.91 \pm 60.39$ & $15.34 \pm 1.67$ & $65.92 \pm 7.03$ \\
\hline Last & $4.88 \pm 0.37$ & $145.17 \pm 9.24$ & $43.77 \pm 2.74$ & $89.82 \pm 5.59$ & $29.83 \pm 2.14$ & $332.02 \pm 9.56$ & $15.45 \pm 0.88$ & $65.55 \pm 5.81$ \\
\hline
\end{tabular}

Note: ${ }^{*} P>0.05$

\section{Post plateletpheresis results from the low frequency group}

In Table 4 6, statistical comparisons from complete blood count data (from the first and the last plateletpheresis) in the low frequency $\operatorname{group}(n=20$; frequency $=6.6 \pm 0.5$ per year) are shown. After plateletpheresis, WBC, RBC and PLT values obeyed normal distribution and all hematological parameters were within the normal range and showed no significant change $(P>0.05)$.

\section{DISCUSSION}

The use of apheresis technique to collect platelets has rapidly increased in recent years. With this increased demand for plateletpheresis, higher donation frequencies are required to meet demand. After 
Table 4 PLT count values for the first and the last screening in low frequent group $\quad(n=54)$

\begin{tabular}{cccccc}
\hline Time & PLT* $^{*}()$ & MPV*( ) & PDW*( ) & PCT*( ) & LPCR*( ) \\
\hline First & $218.05 \pm 41.13$ & $7.92 \pm 1.03$ & $11.78 \pm 1.50$ & $0.17 \pm 0.03$ & $15.43 \pm 6.38$ \\
Last & $211.10 \pm 64.48$ & $7.59 \pm 0.74$ & $11.26 \pm 1.08$ & $0.16 \pm 0.03$ & $13.61 \pm 4.69$ \\
\hline
\end{tabular}

Note: ${ }^{*} P>0.05$

Table 5 WBC count values for the first and the last screening in low frequent group $\quad(n=54)$

\begin{tabular}{|c|c|c|c|c|c|c|c|}
\hline Time & $\mathrm{WBC}^{*}(\mathrm{)}$ & $\mathrm{LA}^{*}()$ & $\mathrm{MA}^{*}()$ & $\mathrm{GA}^{*}()$ & $\mathrm{LR}^{*}(\mathrm{)}$ & $\mathrm{MR}^{*}(\mathrm{)}$ & $\mathrm{GR}^{*}(\mathrm{)}$ \\
\hline First & $5.81 \pm 1.33$ & $1.78 \pm 0.71$ & $0.47 \pm 0.12$ & $3.57 \pm 0.97$ & $31.05 \pm 8.49$ & $7.33 \pm 1.35$ & $61.63 \pm 9.11$ \\
\hline Last & $5.62 \pm 1.27$ & $1.66 \pm 0.55$ & $0.46 \pm 0.13$ & $3.50 \pm 0.78$ & $30.12 \pm 5.63$ & $7.04 \pm 0.99$ & $62.84 \pm 5.74$ \\
\hline
\end{tabular}

Table 6 RBC count values for the first and the last screening in low frequent group $(n=54)$

\begin{tabular}{|c|c|c|c|c|c|c|c|c|}
\hline Time & $\mathrm{RBC}^{*}($ ) & $\operatorname{HGB}^{*}(\quad)$ & $\operatorname{HCT}^{*}()$ & $\mathrm{MCV}^{*}(\mathrm{)}$ & $\mathrm{MCH}^{*}(\mathrm{)}$ & MCHC*( ) & RDWR*( ) & RDWA*( ) \\
\hline First & $5.12 \pm 0.46$ & $146.45 \pm 12.52$ & $44.80 \pm 3.34$ & $88.01 \pm 8.67$ & $28.84 \pm 3.19$ & $327.15 \pm 8.04$ & $15.79 \pm 1.06$ & $64.83 \pm 8.31$ \\
\hline Last & $5.02 \pm 0.48$ & $146.35 \pm 13.06$ & $44.69 \pm 3.69$ & $89.46 \pm 3.69$ & $29.38 \pm 3.19$ & $327.75 \pm 8.33$ & $15.96 \pm 0.68$ & $66.82 \pm 7.84$ \\
\hline
\end{tabular}

plateletpheresis, the recovery to pre-plateletpheresis status is of concern. The effects of plateletpheresis on donor haematological parameters have been studied to some degree, however there still remains conflicting reports on the effect of plateletpheresis on donors. Some studies conclude that even repeated plateletpheresis is safe with no significant adverse effects $^{[6]}$, while others report significant effects on the haematopoietic system ${ }^{[5,7]}$. This study makes a small scale investigation into the impact of frequent multiple plateletpheresis on haematological changes found in blood donors.

Because plateletpheresis leads to the immediate loss of circulating platelets, platelets stored in the spleen are released into blood circulation. At the same time, bone marrow accelerates the production of platelets. The average lifespan of platelets is seven to fourteen days, which is $10 \%$ of the total daily update. It takes about eight to ten days from the original megakaryocyte to release the platelets into the blood. More than half of the platelets entering the blood circulate in the peripheral blood, and the rest are stored in the spleen. After one week, the blood donor's platelet count recovers to the baseline- in the majority of donors. Studies conclude the minimum interval between two plateletpheresis to be at least one week minimum, to allow for enough recovery time. At present, the maximum allowable blood donation for each blood donor in many countries and regions in the world is much wider than the current standard in China. This is due to population, nutritional conditions and physical fitness.

Short-term decreases in platelet count following plateletpheresis have been found to occur without much clinical significance. Moreover, platelet function recovers after one week post plateletpheresis and coagulation after two weeks ${ }^{[8]}$, while double dose plateletpheresis is considered the same ${ }^{[9]}$. This is also consistent with our follow-up test results. As shown in Table 1 and Table 4.

For the screening of pre-plateletpheresis donors, peripheral blood samples are tested for: ALT, blood type, HBsAg and viral serology and nucleic acid testing. The process of plateletpheresis causes the loss of up to $100 \mathrm{~mL}$ of blood per donation ${ }^{[10]}$, owing to adhesion to consumables and the physical effects of centrifugation on the cells. In this study, frequent group plateletpheresis donors' red cell losses may exceed their whole blood donation per year. The slowest recovery for red cells in the peripheral blood, from frequent multiple plateletpheresis donations is currently believed to be a minimum interval of two weeks, and a donation of no more than twenty-four times a year is recommended. This is inconsistent with the results of our follow-up tests however, which may be related to the follow-up period and number of samples. As shown in Table 3 and Table 6.

This test used advanced blood cell separators and the latest software programs to collect platelets and screen for WBC, RBC and PLT before and after plateletpheresis $^{[11-12]}$. The results showed that donors who underwent plateletpheresis in both the high and low frequent groups had no statistical changes in complete blood count values. This result suggests that frequent multiple plateletpheresis is a safe procedure. However, the number of donors who continuously donated in this study was small, and the sample size for future research needs to be further expanded. In conclusion, frequent and multiple plateletpheresis has no effects on complete blood count values, and is absent of adverse effects on donors.

\section{Acknowledgements and funding}

This research was supported by the grant of Science and Technology Commission Project of $\mathrm{Yu}-$ 
zhong District of Chongqing (20140130). We thank all colleagues and donors for their collaboration and assistance.

\section{References}

[1] Gao M, Huang R, Tang H, et al. A Chinese perspective on factors and molecular mechanisms that influence the curative effects of platelet transfusion[J]. Asia-pacifie Journal of Blood Types and Gene,2017, 1(1):11-16

[2] Tuan HT, Hock LS, Abdullah ZW. Haemostatic parameters, platelet activation markers, and platelet indices among regular plateletpheresis donors[J]. Journal of Taibah University Medical Sciences, 2018,13(2):180-187

[3] Schrezenmeier H, Seifried E. Buffy-coat-derived pooled platelet concentrates and apheresis platelet concentrates: which product type should be preferred?[J]. Vox Sang, 2010, 99(1):1-15

[4] Daurat A, Roger C, Gris JC, et al. Apheresis platelets are more frequently associated with adverse reactions than pooled platelets both in recipients and in donors: a study from French hemovigilance data[J]. Transfusion, 2016,56(6):1295-1303

[5] Das SS, Chaudhary R, Verma SK, et al. Pre and post donation haematological values in healthy donors undergoing plateletpheresis with five different systems[J]. Blood Transfus, 2009,7(3):188-192
[6] Katz L, Palmer K, Mcdonnell E, et al. Frequent plateletpheresis does not clinically significantly decrease platelet counts in donors[J]. Transfusion, 2007,47(9):1601-1606

[7] Lazarus EF, Browning J, Norman J, et al. Sustained decreases in platelet count associated with multiple, regular plateletpheresis donations[J]. Transfusion, 2001,41(6):756-761

[8] Zhou Q, Yu X, Cai Y, et al. Changes in pre and post donation platelet function in plateletpheresis donors[J]. Transfusion Clinique et Biologique, 2017,24(4):417-421

[9] Makroo RN, Fadadu D, Agrawal S, et al. Double dose plateletpheresis: a savior to shrinking donor pool and platelet inventory management[J]. Indian Journal of Hematology and Blood Transfusion, 2018,34(4):691-696

[10] Page EA, Coppock JE, Harrison JF. Study of Iron stores in regular plateletpheresis donors[J]. Transfus Med, 2010,20(1):22-29

[11] Strasser EF, Weidinger T, Zimmermann RA, et al. Recovery of white blood cells and platelets from leukoreduction system chambers of Trima Accel and COBE Spectra plateletpheresis devices[J]. Transfusion, 2007, 47(10):1943-1944

[12] Thokala RP, Radhakrishnan K, Anandan A, et al. Recovery of platelet count among apheresis platelet donors[J]. $J$ Clin Diagn Res, 2016,10(12):C1-C4

(Received 29 October 2018, Revised 26 November 2018, Accepted 13 December 2018) 